\title{
HLA-A, B (Class I) and HLA-DR, DQ (Class II) Antigens in Turkish Patients with Recurrent Aphthous Ulceration and Behçet's Disease
}

\author{
Filiz Namdar Pekiner ${ }^{\mathrm{a}}$ Emre Aytugar $^{\mathrm{a}} \quad$ Gülderen Yanikkaya Demirel ${ }^{\mathrm{b}}$ \\ M. Oğuz Borahan ${ }^{a}$ \\ ${ }^{a}$ Department of Oral Diagnosis and Radiology, Faculty of Dentistry, Marmara University Istanbul, and \\ ${ }^{\mathrm{b}}$ Immunology Section, Department of Medical Microbiology, Yeditepe University, Istanbul, Turkey
}

\section{Key Words}

Recurrent aphthous ulceration - Behçet's disease $\cdot$ HLA

class I. HLA class II - Antigen

\begin{abstract}
Objective: The aims of the present study were to typify the human leukocyte antigen system (HLA)-A, B (class I) and HLA-DR, DQ (class II) antigens and to assess the frequency of the presence of these antigens in the Turkish population with recurrent aphthous ulceration (RAU) and Behçet's disease (BD) compared to healthy subjects. Subjects and Methods: Thirty patients with RAU, 30 with $B D$, and 15 healthy subjects were included in the study. HLA typing was performed by serology with commercial kits for HLA class I and II (One Lambda, Canoga Park, Calif., USA). Results: The HLAA23 frequency was $26.7 \%$ in the RAU patients, which was significantly higher than the $3.3 \%$ frequency in the patients with BD $(p<0.05)$. The HLA-A24 frequency was $33.3 \%$ in the RAU patient group, which was significantly higher $(\mathrm{p}<0.05)$ than the frequency in the healthy subjects (6.7\%). Significantly higher frequencies (46.7\%) of HLA-A30 were found in the healthy subjects compared to the BD (13.3\%) and RAU (3.3\%) patients ( $p<0.05$ and $p<0.01$, respectively). A higher frequency of HLA-B13 was observed in the RAU (23.3\%) patients compared to the BD $(0 \%)$ patients $(p<0.01)$. A decrease was observed in HLA-DR10 and HLA-DR17 in the RAU
\end{abstract}

patients $(p<0.05)$, while a higher frequency of HLA-DR10 was observed in the BD patients compared to the RAU patients $(p<0.01)$. Conclusions: These results showed that RAU and BD were not in the same spectrum and the involvement of other genetic and/or environmental factors might be responsible for the development of these diseases and/ or disease progression.

Copyright $\odot 2013$ S. Karger AG, Basel

\section{Introduction}

Recurrent aphthous ulceration (RAU), also known as aphthous stomatitis or canker sores, is the most common inflammatory disease of oral mucosa in children and adults, affecting an estimated $20-25 \%$ of the world's population [1-4]. The disorder is characterized by the appearance of painful and recurrent, single or multiple ulcerations that preferentially affect the nonkeratinized oral mucosa $[4,5]$. Although RAU is global in distribution, it remains one of the least understood diseases of the oral cavity. Patients with RAU tend to be otherwise healthy, so that even in those with severe disease, the process is generally limited to the oral mucosa [1]. Important considerations regarding the differential diagnosis include complex aphthous stomatitis and variants related to systemic diseases, such as those associated with hematologic, hor-

\begin{tabular}{|c|c|}
\hline KARGER & $\begin{array}{l}\text { (c) } 2013 \text { S. Karger AG, Basel } \\
1011-7571 / 13 / 0225-0464 \$ 38.00 / 0\end{array}$ \\
\hline $\begin{array}{l}\text { E-Mail karger@karger.com } \\
\text { www.karger.com/mpp }\end{array}$ & $\begin{array}{l}\text { This is an Open Access article licensed under the terms } \\
\text { of the Creative Commons Attribution-NonCommercial- } \\
\text { NoDerivs } 3.0 \text { License (www.karger.com/OA-license), appli- } \\
\text { cable to the online version of the article only. Distribution } \\
\text { for non-commercial purposes only. }\end{array}$ \\
\hline
\end{tabular}

Dr. Filiz Namdar Pekiner

Department of Oral Diagnosis and Radiology, Faculty of Dentistry

Marmara University, Guzelbahce Buyukciftlik Sok. No: 6

TR-34365 Nisantasi-Istanbul (Turkey)

E-Mail fpekiner@yahoo.com 
monal, nutritional and gastrointestinal alterations [4]. In view of these factors, the clinician needs a detailed history of the patient and a clinical examination is necessary in order to diagnose the clinical conditions that involve RAU and other systems, such as Behçet's disease (BD) $[4,5]$.

$\mathrm{BD}$ is a multisystem inflammatory disease with a wide variety of manifestations, including oral and genital ulcers, skin lesions, ocular inflammation, arthritis, cardiovascular disease, and involvement of the central nervous system [6]. In $\mathrm{BD}$, the majority of patients experience RAU as the most common major symptom, and it is often the initial feature. However, it is impossible to predict if a patient with RAU will develop $\mathrm{BD}$, and there is no reliable test to exclude BD. Therefore, RAU in BD presents a diagnostic problem. The etiology and pathogenesis of $\mathrm{BD}$ are still uncertain; the onset of $\mathrm{BD}$ is believed to be triggered by the involvement of some external environmental factors in individuals with a particular genetic makeup [7]. A strong association of BD with HLA-B51 (one of the split antigens of HLA-B5) has been confirmed in many different ethnic groups from the Middle East to the Far East, and about $40-80 \%$ of patients with BD in these populations possess HLA-B5 (B51) [7]. Ohno et al. [8] hypothesized that genes susceptible to BD closely linked to HLA-B51 might have been spread by the old nomadic or Turkish tribes via the ancient Silk Route. The association of major histocompability complex class II antigens with $\mathrm{BD}$ has also been studied in different groups, but the results vary [9-13]. Only a few studies on the association of human leukocyte antigen system (HLA) class II antigens with the mucocutaneous type of $\mathrm{BD}$ have been reported $[14,15]$.

Chung et al. [16] showed that the phenotype frequency of HLA-DRw8 antigen in 16 Chinese patients with the mucocutaneous type of $\mathrm{BD}$ is significantly higher than that in patients with RAU. Jaber et al. [17] observed that there is a close association between HLA-B52 and HLA-B44 in Israeli Arab youths with RAU but long-term follow-up is needed to determine the relationship between RAU and BD. Özbakir et al. [18] found a significantly higher frequency of HLA-DR4 antigen in patients with RAU than in patients with BD. In the present study, HLA-A, B (class I) and HLA-DR, DQ (class II) antigens were analyzed in a group of 30 Turkish RAU and 30 Turkish BD patients.

\section{Subjects and Methods}

\section{Selection of the Study Group}

Thirty patients ( 12 men and 18 women) with RAU, another 30 patients (15 men and 15 women) with BD, and 15 healthy subjects
(9 men and 6 women) participated in the study. The diagnosis of RAU was made according to the criteria of Lehner [19], and all were diagnosed with minor or major RAU in the Department of Oral Diagnosis and Radiology, Faculty of Dentistry, Marmara University. The RAU patients had aphthous ulcerations that recurred at least once in 2 months during a 1 -year period prior to this study. Subjects with herpetiform ulceration or those in whom the diagnosis was not clear were excluded from the study. Thirty patients with BD who fulfilled the International Study Group criteria were selected from the Rheumatology Out-Patient Clinic by an expert rheumatologist (Melek Özer) [20]. For the patients with RAU and BD, the duration of aphthous ulcers was recorded. Fifteen healthy subjects comprising faculty personnel participated after having given informed consent. All healthy subjects were questioned about the absence of recurrent oral aphthous ulcers or other BD-related manifestations or a family history of BD. Similarly, the RAU and BD patients were requested to sign a written informed consent. The study was carried out according to the recommendations of the Helsinki Declaration. The study protocol was approved by the Local Committee of Research and Ethics of Marmara University. All patients and healthy subjects were of Turkish origin and not related to one another.

\section{Analysis of HLA Typing}

For HLA, serology typing was performed using commercial kits for HLA class I and II (One Lambda, Canoga Park, Calif., USA). For HLA-A, B (major histocompability complex I) and HLA-DR, DQ (major histocompability complex II) Terasaki HLA tissue typing trays were used.

Mononuclear cells were obtained from whole blood samples drawn into heparin tubes by density centrifugation with Lympho-Kwik ${ }^{\circledR}$ Isolation Reagents (One Lambda). T and B lymphocytes were separated using EasySep ${ }^{\circledR}$ Cell Isolation System (Stem Cell Technologies, Canada). Isolated cells were pipetted into 96-well plates. Before seeding, the cells' viability was controlled by staining with trypan blue solution (Sigma, USA) and counting viable cells under light microscopy (Leica, Germany). Cells that had more than $90 \%$ viability were processed. The cell concentration was adjusted to $2 \times 10^{6}$ cells $/ \mathrm{ml}$. Cells were incubated in the presence of a complement binding antibody (One Lambda). After this incubation period, the complement was added into the wells. FluoroQuench $^{\mathrm{TM}}$ (One Lambda) was used to stain and fix lymphocytes in the microcytotoxicity assay since a quenching reagent can significantly reduce background fluorescence. Ethylenediaminetetraacetic acid was used to stop complement-dependent cell lysis. Ethidium bromide was used to stain dead cells while acridine orange was used to stain live cells. Live lymphocytes (nonreactive) stained green, while for positive reactions lymphocytes stained orange/red. For each patient sample, amounts of live and dead cells were evaluated with a fluorescence microscope (Olympus, Japan). Each plate was evaluated by two independent observers (G.Y.D., Mustafa Aslan).

\section{Statistical Analysis}

The data were analyzed with NCSS (Number Cruncher Statistical System) 2007 and PASS 2008 Statistical Software (Kaysville, Utah, USA). The age, gender, clinical characteristics, and HLA typing between patients with BD or RAU and controls were analyzed by means of the $\chi^{2}$ test. $p$ values $<0.05$ were interpreted as significant, and the level of confidence intervals was $95 \%$. 
Table 1. Frequencies of HLA-A and B and HLA-DR and DQ antigens in patients with BD and RAU and in healthy controls

\begin{tabular}{|c|c|c|c|c|c|}
\hline \multirow[t]{2}{*}{ HLA antigens } & \multicolumn{3}{|l|}{ Patients } & \multirow{2}{*}{$\mathrm{p}$} & \multirow[t]{2}{*}{ Odds ratio $(95 \% \mathrm{CI})$} \\
\hline & $\begin{array}{l}\text { with BD } \\
(\mathrm{n}=30)\end{array}$ & $\begin{array}{l}\text { with RAU } \\
(\mathrm{n}=30)\end{array}$ & $\begin{array}{l}\text { Healthy } \\
\text { controls }(n=15)\end{array}$ & & \\
\hline $\mathrm{A} 23$ & $1(3.3)$ & $8(26.7)$ & & $0.026^{*}$ & $0.095(0.011-0.815)$ \\
\hline A24 & & $10(33.3)$ & $1(6.7)$ & $0.049^{*}$ & $7.000(0.802-61.070)$ \\
\hline A30 & $4(13.3)$ & & $7(46.7)$ & $0.026^{*}$ & $0.176(0.041-0.759)$ \\
\hline A30 & & $1(3.3)$ & $7(46.7)$ & $0.001^{* *}$ & $0.039(0.004-0.369)$ \\
\hline B13 & $0(0)$ & $7(23.3)$ & & $0.011^{*}$ & $2.304(1.694-3.134)$ \\
\hline DR10 & $10(33.3)$ & $1(3.3)$ & & $0.006^{*}$ & $14.500(1.718-122.395)$ \\
\hline DR10 & & $1(3.3)$ & $5(33.3)$ & $0.012^{*}$ & $0.069(0.007-0.664)$ \\
\hline DR17 & & $1(3.3)$ & $5(33.3)$ & $0.012^{*}$ & $0.069(0.007-0.664)$ \\
\hline
\end{tabular}

The values for patients and controls are given as number with the percentage in parentheses. Fisher's exact test. ${ }^{*} \mathrm{p}<0.05$; ${ }^{* *} \mathrm{p}<0.01$.

\section{Results}

The mean age of the patients with RAU was 37.03 \pm 13.8 years (range 17-64) while that of those with BD was $38.06 \pm 8.55$ years (range 15-54) and that of the healthy control subjects was $30.26 \pm 8.98$ years (range $23-51$ ). The differences were not statistically significant.

Frequencies of HLA-A, B (class I) and HLA-DR, DQ (class II) are shown in table 1. The HLA-A23 antigen frequency was $26.7 \%$ in the RAU patient group compared to the $3.3 \%$ in the BD patients ( $\mathrm{p}=0.05)$. The HLA-A24 antigen frequency was $33.3 \%$ in the RAU patient group compared to the $6.7 \%$ in the healthy subjects $(\mathrm{p}<0.05)$. A significantly higher frequency of HLA-A30 was found in the healthy subjects compared to the RAU patients $(\mathrm{p}<0.01)$. A higher frequency of HLA-B13 was observed in the RAU patients $(0 \%)$ compared to the BD patients $(23.3 \%$; $\mathrm{p}<$ 0.05). A lower frequency of HLA-DR10 and HLA-DR17 in the RAU patients was observed compared to the healthy subjects $(\mathrm{p}<0.05)$. A statistically higher frequency of HLADR10 was observed in the BD patients compared to the RAU patients $(\mathrm{p}<0.01)$. A significantly higher frequency of HLA-A30 was found in the healthy subjects compared to the $\mathrm{BD}$ patients $(\mathrm{p}<0.05)$. The differences among all the other HLA-A, B (class I) and HLA-DR, DQ (class II) antigens were not statistically significant in the patients with RAU and BD and the healthy subjects (table 1).

\section{Discussion}

Although the immunopathogenic mechanisms of RAU and $\mathrm{BD}$ are not clear, infectious agents, immune mechanisms and genetic factors are thought to be in- volved in the onset of the diseases [7,21], immunogenetic factors seem to play an important role in susceptibility and resistance. Nevertheless, the correlation between HLA antigens and pathogenesis is still obscure. It is hard to predict whether RAU will eventually become BD or not, especially because the relationship between RAU and $\mathrm{BD}$ is still controversial $[14,15,18]$.

In this study, HLA-A23 and HLA-B13 had a higher frequency in patients with RAU compared to $\mathrm{BD}$ while HLA-DR10 had a higher frequency in BD patients compared to RAU patients. These findings suggest that RAU and $\mathrm{BD}$ are not in the same spectrum as has been observed in other studies [18, 22-24], and that the involvement of other genetic and/or environmental factors may be responsible for the development and/or progression of these diseases. Nevertheless, Lehner et al. [14] had suggested that BD and RAU may be in the same spectrum; however, Sun et al. [15] observed that there is no significant difference in the frequencies of HLA-DR and DQ antigens between patients with $\mathrm{BD}$ and those with RAU. Our findings seem to indicate that HLA-A30 might be considered a marker of low specificity of RAU and BD in Turkish people and that it may be a protector HLA antigen. But this assumption needs to be confirmed by further studies.

BD is strongly associated with HLA-B51 in many different ethnic groups $[8,11,12]$, although it is still unclear whether the susceptibility to BD is influenced by HLAB51 itself or by some other non-HLA antigen $[7,23]$. Our results did not reveal any remarkable influence of HLAB51 on the severity of BD. However, this does not answer the question of whether the susceptibility antigen related to the disease is the HLA-B51 itself or another non-HLA antigen in linkage disequilibrium with the HLA-B51. 
Despite the well-established association of HLA-B51 with BD in many ethnic groups, different results of the association between HLA class II antigen and BD-RAU have been reported $[15,16,18]$. According to Lehner et al. [14], the immunogenetic basis of BD and RAU based on the HLA-A and B loci has been extended to the HLADR locus. Therefore, in this study, we investigated whether HLA class II antigens are associated with BD and RAU. In our analysis of HLA class II antigen in BD and RAU, the frequency of HLA-DR10 and HLA-DR17 was found to be relatively low in Turkish patients with RAU compared to healthy control subjects and the frequency of HLA-DR10 was found to be relatively low in Turkish patients with RAU compared to those with BD (table 1). These results suggest that some specific HLA-DR antigens may play important roles in the development of $\mathrm{BD}$ and RAU. On the other hand, according to Pirim et al. [7], in their genotyping of HLA class II alleles, HLA-DRB104 and HLA-DRB107 were the predominant alleles in BD Turkish patients. An association of BD with HLA-DRw52 in Japanese people [8], with HLA-DR7, HLA-DRw52, and HLA-DRw53 in British people [14] and with HLADRw52 in Italian people has been reported [25]. Sun et al. [6] demonstrated a strong association of the HLA-DRw8 antigen as well as HLA-DRw8/DQw1, DRw8/DQw5(w1), DRw12(5)/DQw1, DRw12(5)/DQw6(w1), and DRw52/ DQw1 haplotypes with BD in Chinese people. In addition, they showed no significant association in the frequencies of HLA-DR and DQ antigens in Chinese patients with $\mathrm{BD}$ compared to those in healthy control groups [15]. In their genotyping of HLA class II alleles, Pirim et al. [7] found that HLA-DRB104 and HLADRB107 were the predominant alleles in BD patients. Although there were different HLA class allele distributions within the groups, the results were not significant. They showed that there is a lack of an association of class II alleles with BD. Kera et al. [26] reported similar results; the frequency of HLA-DR5 was found to be relatively high in $\mathrm{BD}$ patients compared to that in healthy controls but was not statistically significant. In contrast, in another study by Balboni et al. [27], the same HLA-DR5 and the DQ3 frequency was found as well when Italian patients with BD were studied. Similar to our study, these authors [26, 27] analyzed HLA class II association with BD in Italian patients only by the serological method and reported a significant increase of the DR5-DQ3 haplotype. Lehner et al. [14] suggested that the immunogenetic basis of $B D$ and RAU based on the HLA-A and B loci be extended to the HLA-DR locus. Gallina et al. [28] found a statistically increased value for the HLA-DR7 frequency for a sample of

HLA-A, B and HLA-DR, DQ in Turkish

Patients with RAU and BD
26 Sicilian patients with RAU. The frequency of HLADR7 antigen increased significantly in RAU-affected persons, whereas the B5 antigen frequency decreased significantly. In another study [18], data showed an increased frequency of HLA-DR4 antigen in patients with RAU compared to that in patients with BD. In our analysis of HLA class II antigen in BD and RAU, the frequency of HLA-DR10 and HLA-DR17 was found to be relatively low in Turkish patients with RAU compared to healthy control subjects, and the frequency of HLA-DR10 was found to be relatively low in Turkish patients with RAU compared to those with $\mathrm{BD}$ (table 1). These results suggest that some specific HLA-DR antigens may play important roles in the development of BD and RAU. Numerous studies found that the HLA class II antigen frequencies for BD and RAU were different from those found in our study probably because populations of different ethnic origins were studied (British, Italian, or Chinese).

Several reports from different countries showed a genetic correlation of RAU with HLA antigens, but the results of these studies are controversial $[6,14,28,29]$. In our study, HLA-A24 and B13 had a higher frequency for RAU, while HLA-DR10 and DR17 had a lower frequency (table 1). In agreement with other studies [8, 12], this finding supports the hypothesis that a specific antigen may possibly be responsible for RAU. In an English population, Challacombe et al. [29] found that the HLA-B12 frequency was associated with patients with RAU. In groups of patients of a different ethnic origin, a significant association between HLA-DR2 and RAU was noticed $[9,14]$. Wilhelmsen et al. [30] observed that the HLA frequencies that had the highest rates of patients with RAU were HLA-A33, HLA-B35, and HLA-B81. Gallina et al. [28], with a sample of 26 Sicilian patients with RAU, found a statistically increased value for HLA-DR7 frequency. Sun et al. [6] stated that the HLA-DRw9 frequency can be considered to be a genetic marker for RAU in the Chinese population. Other previous cross-sectional studies from the United Kingdom and Greece have suggested that certain HLA antigens are linked to RAU, notably the class I antigens A2 and B12 [14] and the class II antigen DR5 [9]. In our study, HLA-A24 and B13 were associated with an increased risk of RAU, while HLADR10 and DR17 were associated with a decreased risk (table 1). In agreement with other studies [8, 12], this finding supports the hypothesis that a specific antigen may possibly be responsible for RAU. Finally, further studies could be mapped to the possible genetic factors involved in the genesis of RAU for each population. 


\section{Conclusion}

Our findings showed that BD and RAU were not in the same spectrum; there were no differences of HLA-B51 among BD, RAU, and healthy subjects. The presence of HLA-A30 is useful as a determiner for subjects at risk of developing $\mathrm{BD}$ and RAU. We recommend that a more advanced method such as single specific primer-polymerase chain reaction should be used to obtain more specific results as well. We aim to further our studies with more advanced methods in the near future.

\section{Acknowledgement}

This research was supported by the Marmara University Scientific Research Project Council (project No. SAG-DKR-2004070078). Authors would like to thank Biologist. Mustafa Aslan, $\mathrm{PhD}$ for his technical assistance, Centro Laboratories for providing the use of laboratory equipment, and expert rheumatologist, Dr. Melek Özer.

\section{References}

1 Oh SH, Han EC, Lee JH, et al: Comparison of the clinical features of recurrent aphthous stomatitis and Behçet's disease. Clin Exp Dermatol 2009;34:e208-e212.

-2 Alpsoy E, Donmez L, Onder M, et al: Clinical features and natural course of Behçet's disease in 661 cases: a multicentre study. Br J Dermatol 2007;157:901-906.

3 Karincaoglu Y, Borlu M, Toker SC, et al: Demographic and clinical properties of juvenileonset Behçet's disease: a controlled multicenter study. J Am Acad Dermatol 2008;58: 579-584.

4 Tunes RS, Anjos TC, Martins GB, et al: Prevalence of Behçet's syndrome in patients with recurrent aphthous ulcerations in Brazil. Rheumatol Int 2009;29:875-878.

5 Jurge S, Kuffer R, Scully C, et al: Mucosal disease series. Number VI. Recurrent aphthous stomatitis. Oral Dis 2006;12:1-21.

6 Sun A, Hsieh RP, Chu CT, et al: Some specific human leukocyte antigen (HLA)-DR/ DQ haplotypes are more important than individual HLA-DR and -DQ phenotypes for the development of mucocutaneous type of $\mathrm{Be}$ hçet's disease and for disease shift from recurrent aphthous stomatitis to mucocutaneous type of Behçet's disease. J Oral Pathol Med 2001;30:402-407.

7 Pirim I, Atasoy M, Ikbal M, et al: HLA class I and class II genotyping in patients with Behçet's disease: a regional study of eastern part of Turkey. Tissue Antigens 2004;64:293-297.

8 Ohno S, Ohguchi M, Hirose S, et al: Close association of HLA-B51 with Behçet's disease. Arch Ophthalmol 1986;100:1455-1458.

-9 Albanidou-Farmaki E, Deligiannidis A, Markopoulos AK, et al: HLA haplotypes in recurrent aphthous stomatitis: a mode of inheritance? Int J Immunogenet 2008;35:427-432.

-10 Soto-Vega E, García-Muñoz R, Richaud-Patin Y, et al: Class I and class II MHC polymorphisms in Mexican patients with Behçet's disease. Immunol Lett 2004;93:211-215.
Mizuki N, Meguro A, Tohnai I, et al: Association of major histocompatibility complex class I chain-related gene A and HLA-B alleles with Behçet's disease in Turkey. Jpn J Ophthalmol 2007;51:431-436.

12 Choukri F, Chakib A, Himmich H, et al: HLA$\mathrm{B}^{*} 51$ and $\mathrm{B}^{*} 15$ alleles confer predisposition to Behçet's disease in Moroccan patients. Hum Immunol 2001;62:180-185.

13 Ahn JK, Park YG: Human leukocyte antigen B27 and B51 double-positive Behçet uveitis. Arch Ophthalmol 2007;125:1375-1380.

14 Lehner T, Welsh KI, Batchelor JR: The relationship of HLA-B and DR phenotypes to Behçet's syndrome, recurrent oral ulceration and the class of immune complexes. Immunology 1982;47:581-587.

15 Sun A, Lin SC, Chu CT, et al: HLA-DR and -DQ antigens in Chinese patients with Behçet's disease. J Oral Pathol Med 1993;22:6063.

16 Chung YM, Tsai ST, Liao F, et al: A genetic study of Behçet's disease in Taiwan Chinese. Tissue Antigens 1987;30:68-72.

17 Jaber L, Weinberger A, Klein T, et al: Close association of HLA-B52 and HLA-B44 antigens in Israeli Arab adolescents with recurrent aphthous stomatitis. Arch Otolaryngol Head Neck Surg 2001;127:184-187.

18 Özbakir F, Yazici H, Mat C, et al: HLA antigens in recurrent oral ulceration: evidence against a common disease spectrum with Behçet's syndrome. Clin Exp Rheumatol 1987;5:263-265.

19 Lehner T: Immunologic aspects of recurrent oral ulcers. Oral Surg Oral Med Oral Pathol 1972;33:80.

20 Irschick EU, Philipp S, Shahram F, et al: Investigation of bacterial and viral agents and immune status in Behcet's disease patients from Iran. Int J Rheum Dis 2011;14:298-310.
21 Turgut S, Turgut G, Atalay EÖ, et al: Angiotensin-converting enzyme I/D polymorphism in Behçet's disease. Med Princ Pract 2005; 14:213-216.

-22 Özdemir M, Acar H, Deniz F, et al: HLA-B*51 in patients with recurrent aphthous stomatitis. Acta Derm Venereol 2009;89:202-203.

23 Gül A, Uyar FA, Inanc M, et al: Lack of association of HLA-B51 with a severe disease course in Behçet's disease. Rheumatology 2001;40:668-672.

24 Chang HK, Kim JU, Cheon KS, et al: HLA$\mathrm{B} 51$ and its allelic types in association with Behçet's disease and recurrent aphthous stomatitis in Korea. Clin Exp Rheumatol 2001;19: 31-35.

25 Baricordi OR, Sensi A, Pivetti-Pezzi P, et al: Behçet's disease associated with HLA-B51 and DRw52 antigens in Italians. Hum Immunol 1986;17:297-301.

26 Kera J, Mizuki N, Ota M, et al: Significant associations of HLA*5101 and B*5108, and lack of association of class II alleles with Behçet's disease in Italian patients. Tissue Antigens 1999;54:565-571.

27 Balboni A, Pivetti-Pezzi P, Orlando P, et al: Serological and molecular HLA typing in Italian Behçet's patients: significant association to B51-DR5-DQw3 haplotype. Tissue Antigens 1992;39:141-143.

28 Gallina G, Cumbo V, Messina P, et al: HLA-A, $\mathrm{B}, \mathrm{C}, \mathrm{DR}, \mathrm{MT}$, and $\mathrm{MB}$ antigens in recurrent aphthous stomatitis. Oral Surg Oral Med Oral Pathol 1985;59:364-370.

29 Challacombe SJ, Barkhan P, Lehner T: Haematological features and differentiation of recurrent oral ulceration. Br J Oral Surg 1977; 15:37-48.

30 Wilhelmsen NSW, Weber R, Monteiro F, et al: Correlation between histocompatibility antigens and recurrent aphthous stomatitis in the Brazilian population. Braz J Otorhinolaryngol 2009;75:426-431. 